\title{
Human biomonitoring of essential, nonessential, rare earth, and noble elements in placental tissues
}

\section{Aleksandar Stojsavljević, Marija Rovčanin, Branislav Rovčanin, Željko Miković, Ana Jeremić, Milan Perović, and Dragan Manojlović}

Table S1a. Recovery (R) values for examined trace elements obtained with SRMs by ICP-MS.

\begin{tabular}{|c|c|c|c|c|c|c|c|}
\hline \multirow[t]{2}{*}{ Element } & \multirow{2}{*}{$\begin{array}{l}\text { Internal } \\
\text { standard }\end{array}$} & \multicolumn{2}{|c|}{ Whole blood Level-1 } & \multirow[t]{2}{*}{$\mathrm{R}(\%)$} & \multicolumn{2}{|c|}{ Whole blood Level-2 } & \multirow[t]{2}{*}{$\mathrm{R}(\%)$} \\
\hline & & Measured & Declared & & Measured & Declared & \\
\hline $\mathrm{Al}(\mu \mathrm{g} / \mathrm{L})$ & $\mathrm{Li}$ & 11.0 & 11.2 & 98.2 & 72.1 & 71.8 & 100.4 \\
\hline $\mathrm{Sc}(\mu \mathrm{g} / \mathrm{L})$ & $\mathrm{Li}$ & 0.050 & 0.046 & 108.7 & 0.059 & 0.055 & 105.4 \\
\hline $\mathrm{Ti}(\mu \mathrm{g} / \mathrm{L})$ & $\mathrm{Ge}$ & 15.96 & 14 & 114 & 17 & 18 & 94.4 \\
\hline $\mathrm{Cr}(\mu \mathrm{g} / \mathrm{L})$ & $\mathrm{Ge}$ & 0.79 & 0.82 & 96.3 & 12.1 & 12.4 & 97.6 \\
\hline $\operatorname{Mn}(\mu \mathrm{g} / \mathrm{L})$ & In & 19.0 & 20.1 & 94.5 & 33.2 & 32.7 & 101.5 \\
\hline $\operatorname{Co}(\mu \mathrm{g} / \mathrm{L})$ & $\mathrm{Ge}$ & 0.25 & 0.23 & 108.6 & 6.1 & 6.0 & 101.7 \\
\hline $\mathrm{Ni}(\mu \mathrm{g} / \mathrm{L})$ & In & 1.24 & 1.41 & 88.1 & 17.8 & 18.5 & 96.2 \\
\hline $\mathrm{Cu}(\mu \mathrm{g} / \mathrm{L})$ & In & 643 & 645 & 99.7 & 1245 & 1248 & 99.8 \\
\hline $\mathrm{Zn}(\mathrm{mg} / \mathrm{L})$ & In & 4.79 & 4.81 & 99.5 & 8.3 & 8.406 & 98.7 \\
\hline $\mathrm{Ga}(\mu \mathrm{g} / \mathrm{L})$ & $\mathrm{Ge}$ & 0.020 & 0.022 & 90.9 & 0.031 & 0.030 & 103.3 \\
\hline As $(\mu \mathrm{g} / \mathrm{L})$ & $\mathrm{Ge}$ & 6.39 & 6.32 & 101.1 & 15.3 & 16.3 & 93.9 \\
\hline $\operatorname{Se}(\mu \mathrm{g} / \mathrm{L})$ & In & 101 & 104 & 97.1 & 267 & 264 & 101.1 \\
\hline $\mathrm{Rb}(\mathrm{mg} / \mathrm{L})$ & In & 1.50 & 1.47 & 102.0 & 1.41 & 1.44 & 97.9 \\
\hline $\mathrm{Sr}(\mu \mathrm{g} / \mathrm{L})$ & In & 25 & 24 & 104.2 & 23 & 24 & 95.8 \\
\hline $\mathrm{Y}(\mu \mathrm{g} / \mathrm{L})$ & $\mathrm{Ge}$ & 0.085 & 0.092 & 92.4 & 121 & 123 & 98.4 \\
\hline Mo $(\mu \mathrm{g} / \mathrm{L})$ & $\mathrm{Ge}$ & 0.83 & 0.80 & 103.8 & 6.8 & 6.6 & 103 \\
\hline $\mathrm{Ru}(\mu \mathrm{g} / \mathrm{L})$ & In & 0.046 & $<0.050$ & 92 & 0.046 & $<0.050$ & 92 \\
\hline $\mathrm{Rh}(\mu \mathrm{g} / \mathrm{L})$ & In & 0.018 & $<0.020$ & 90 & 0.018 & $<0.020$ & 90 \\
\hline $\mathrm{Pd}(\mu \mathrm{g} / \mathrm{L})$ & In & 0.009 & $<0.010$ & 90 & 0.009 & $<0.010$ & 90 \\
\hline
\end{tabular}




\begin{tabular}{lccccccc}
\hline $\mathrm{Cd}(\mu \mathrm{g} / \mathrm{L})$ & $\mathrm{Ge}$ & 0.68 & 0.67 & 101.5 & 6.6 & 6.5 & 101.5 \\
$\mathrm{Ba}(\mu \mathrm{g} / \mathrm{L})$ & $\mathrm{Ge}$ & 540 & 542 & 99.6 & 541 & 535 & 101.1 \\
$\mathrm{Ce}(\mu \mathrm{g} / \mathrm{L})$ & In & 0.119 & 0.122 & 97.5 & 0.120 & 0.124 & 96.8 \\
$\mathrm{Pr}(\mu \mathrm{g} / \mathrm{L})$ & $\mathrm{In}$ & 0.022 & 0.024 & 91.7 & 0.022 & 0.025 & 88 \\
$\mathrm{Nd}(\mu \mathrm{g} / \mathrm{L})$ & In & 0.097 & 0.096 & 101 & 0.098 & 0.099 & 98.9 \\
$\mathrm{Sm}(\mu \mathrm{g} / \mathrm{L})$ & In & 0.029 & 0.027 & 107.4 & 0.022 & 0.024 & 91.7 \\
$\mathrm{Eu}(\mu \mathrm{g} / \mathrm{L})$ & In & 0.010 & 0.011 & 90.9 & 0.010 & 0.011 & 90.9 \\
$\mathrm{Gd}(\mu \mathrm{g} / \mathrm{L})$ & In & 0.011 & 0.012 & 91.7 & 0.011 & 0.012 & 91.7 \\
$\mathrm{Er}(\mu \mathrm{g} / \mathrm{L})$ & In & 0.008 & 0.009 & 88.9 & 0.011 & 0.010 & 101 \\
$\mathrm{Yb}(\mu \mathrm{g} / \mathrm{L})$ & In & 0.008 & 0.009 & 88.9 & 0.011 & 0.010 & 101 \\
$\mathrm{Hg}(\mu \mathrm{g} / \mathrm{L})$ & In & 1.94 & 1.97 & 98.5 & 14.3 & 15.2 & 94.1 \\
$\mathrm{~Pb}(\mu \mathrm{g} / \mathrm{L})$ & In & 14.6 & 14.8 & 98.6 & 340 & 336 & 101.2 \\
$\mathrm{Th}(\mu \mathrm{g} / \mathrm{L})$ & In & 0.011 & 0.012 & 91.6 & 0.010 & 0.011 & 90.9 \\
$\mathrm{U}(\mu \mathrm{g} / \mathrm{L})$ & In & 0.17 & 0.18 & 94.4 & 0.11 & 0.12 & 91.7 \\
\hline
\end{tabular}


Table S1b. The standard addition recovery $(\mathrm{R})$ experiment for selected trace elements in placental tissues.

\begin{tabular}{cccc}
\hline Element & Internal standard & \multicolumn{2}{c}{$\mathrm{R}(\%)$} \\
\hline $\mathrm{Tb}$ & $\mathrm{In}$ & $97.8^{*}$ & $101.6^{* *}$ \\
$\mathrm{Dy}$ & $\mathrm{In}$ & $99.8^{*}$ & $99.5^{* *}$ \\
$\mathrm{Ho}$ & $\mathrm{In}$ & $101.2^{*}$ & $97.8^{* *}$ \\
$\mathrm{Lu}$ & $\mathrm{In}$ & $98.4^{*}$ & $103^{* *}$ \\
$\mathrm{Re}$ & $\mathrm{In}$ & $99.2^{*}$ & $98.1^{* *}$ \\
$\mathrm{Os}$ & $\mathrm{In}$ & $97.9^{*}$ & $99.1^{* *}$ \\
$\mathrm{Ir}$ & $\mathrm{In}$ & $102.1^{*}$ & $101.9 * *$ \\
$\mathrm{Pt}$ & $\mathrm{In}$ & $97.7^{*}$ & $98.9 * *$ \\
$\mathrm{Au}$ & $\mathrm{In}$ & $98.6^{*}$ & $97.1^{* *}$ \\
$\mathrm{Bi}$ & $\mathrm{In}$ & $98.9^{*}$ & $99.6^{* *}$ \\
\hline
\end{tabular}

*spiked with $10 \mu \mathrm{g} / \mathrm{L} ;{ }^{* *}$ spiked with $20 \mu \mathrm{g} / \mathrm{L}$. 